\title{
EMG-based Motion Intention Recognition for Controlling a Powered Knee Orthosis
}

\author{
Pedro Nuno Fernandes, Joana \\ Figueiredo, Luis Moreira, Paulo Félix, \\ Ana Correia \\ Center for MicroElectroMechanical \\ Systems (CMEMS) \\ University of Minho \\ Guimarães, Portugal \\ pnlfernandes@gmail.com, \{id6003, \\ a74999, a61767, \\ a71473\}@alunos.uminho.pt
}

\author{
Cristina P. Santos \\ Center for MicroElectroMechanical \\ Systems (CMEMS)-University of Minho, \\ Artificial Intelligence and Computer \\ Science Laboratory (LIACC/FEUP)- \\ University of Porto \\ Portugal \\ cristina@dei.uminho.pt
}

\begin{abstract}
Powered assistive devices have been playing a major role in gait rehabilitation. This work aims to develop a useroriented assistive strategy with an EMG-based control using a powered knee orthosis (PKO) to provide assistive commands according to the user's motion intention tracked by electromyography (EMG) signals. To achieve this goal, the work first comprised the development of a wired EMG acquisition system, the study and implementation of a knee joint torque estimation method, and the development of a real-time controller, which uses the estimated torque as the reference actuator's torque to provide user-oriented assistance in walking. We used a proportional gain method to estimate the knee torque, which required a calibration procedure, allowing to determine the relation between the EMG signal and the actuator's torque. The EMG-based control was validated with two subjects walking in a treadmill. The EMG-based control performed as expected since it proved to be functional and time-effective when assisting the user's movements in walking at different walking speeds. Findings show that the developed assistive strategy can effectively follow the user's motion intention and has the potential for gait rehabilitation of patients with residual muscular strength.
\end{abstract}

Keywords- user-oriented assistive strategy, EMG sensors, motion intention recognition, control strategies, assistive orthosis

\section{INTRODUCTION}

Lower-limb orthoses are Powered Assistive Devices (PADs) act in parallel with the user lower limb to increase motor function, build up joint strength and rehabilitate through task-oriented and repetitive gait training [1], [2]. PADs could implement user-oriented assistive strategies that take into account the body condition of the user with information from biomedical sensors. In recent years, more focus has been given to use Electromyographic (EMG)-based assistive strategies into PAD to provide a functional gait training to encourage muscular effort, and avoiding muscle atrophy [3]-[6]. EMG signals track the muscle activity and consequently, may be applied to analyze muscle disabilities or the progress in gait rehabilitation. The major advantage highlighted regarding these control systems is their ability to predict the user's intended motion, as long as the muscles are not too weak to move.

This work has been supported in part by the Fundação para a Ciência e Tecnologia (FCT) with the Reference Scholarship under Grant SFRH/BD/108309/2015, the reference project UID/EEA/04436/2019, by FEDER funds through the COMPETE 2020 - Programa Operacional Competitividade e Internacionalização (POCI) - with the reference Project POCI-01-0145-FEDER-006941; and the LIACC Project UID/CEC/00027/2019; and with national funds from FCT project SmartOsunder Grant NORTE-01-0145-FEDER-030386.
With respect to knee orthoses, distinct EMG-based assistive strategies have been proposed. In general, the torque of the PAD's actuator should timely match the user's knee torque, estimated from the acquired superficial EMG signals, by means of a torque control. Most studies have applied a complex musculoskeletal model for the knee torque estimation from EMG signals. For instance, Fleisher et al. [5] and Hassani et. al. [7] reported the use of a modified Hill-type. Although the effectiveness of this model, it involves complex methods and several calibration procedures to determine a lot of parameters. On the other hand, simpler approaches based on proportional methods have also been addressed. Kawamoto et al. [8] proposed a simple calibration method for HAL-3 exoskeleton, that only requires finding two proportional gains that relate the recorded extension and flexion EMG signals with the torque generated at the knee. Given the constraints in the field of gait rehabilitation and assistance, this method stands for its simplicity, real-time effectiveness and easy calibration.

This work aims to implement and validate a user-oriented assistive strategy with EMG-based control architecture for controlling a Powered Knee Orthosis (PKO) according to the user's motion intention. For the knee torque estimation, the proportional approach proposed by Kawamoto et al. [8] was adopted. This study covers the complete design of a method for knee joint torque estimation, simple in nature and with an intuitive and effective calibration procedure, and the implementation of a new real-time user-oriented assistive controller for the PKO system. To accomplish this goal, we developed a low-cost, superficial (surface electrodes), wiredEMG signal acquisition system, adjustable for distinct subjects. The proposed EMG-based assistive strategy was validated with two healthy participants walking in a treadmill and the effectiveness of the controller based on user's intentions was inspected and compared with other intention-based strategy, namely, User-PKO interaction torque strategy similar to the one described in [9]. We designed and implemented the EMGbased control such that it simultaneously follows the user's motion intention and may be applied for patients with moderate muscular weakness. Consequently, it can be introduced in an earlier stage of gait rehabilitation than the User-PKO interaction torque strategy, which follows the interaction between user and PKO with higher muscular strength from the user. 


\section{Methods}

To achieve an EMG-based assistive strategy, we developed the system presented in Fig.1. We used the STM32F4 Discovery Board running at $168 \mathrm{MHz}$ to process the acquired EMG signal, to implement the user's knee torque estimation method, and to perform the EMG-based control.

\section{A. Knee Muscles Selection}

Throughout literature, it cannot be found consistency in the number of muscles chosen to control PAD. The study [10] uses two extensor muscles and one flexor muscle, in [6] and [11] used one flexor and one extensor muscles, [12] used three flexor and three extensor muscles, and [13] selected four flexor and four extensor muscles. However, there is consistency in the type of muscles chosen as they are all muscles located on the thigh segment. We used two flexor (Semitendinosus and Semimembranosus) and two extensor (Vastus Lateralis and Vastus Medialis) muscles located in the thigh (Fig.1.A), as [13] shows that the more muscles took into account the more realistic and feasible the control will be, although a trade-off between performance and simplicity of the control strategy was preserved. Based on the study [12], these are the muscles that contribute more to the knee joint movement during gait.

\section{B. EMG Signal Acquisition \& Processing}

In general, the raw EMG signal measured from the surface electrodes has a peak-to-peak magnitude range from 0 to $10 \mathrm{mV}$ and response frequency limited from 0 to $500 \mathrm{~Hz}$, with dominant frequency from 50 to $150 \mathrm{~Hz}$ [14]. In order to infer the muscle's activity, the raw EMG signal must be properly acquired and processed in the developed EMG acquisition system. The first block includes an instrumentation amplifier to obtain the electric potential of the muscle's activity, with an amplification gain factor of 50 to allow the signal to be amplified without significant noise. Then, an active $2^{\text {nd }}$ order bandpass (cut-off frequency from 20 to $500 \mathrm{~Hz}$ ) and a notch (cut-off frequency of $50 \mathrm{~Hz}$ ) were applied to attenuate the effect of motions artifacts and ambient noise, respectively. Next, we introduced an amplifier with a variable gain factor from 1 to 1000 to increase the signal resolution, allowing readability. Then, an offset voltage adjustment circuit was implemented to adjust the EMG signal to an operable voltage range by the ADC of the used digital processing unit. Afterward, we implemented a full wave rectifier (depending on the state of the switch) for signal rectification. The final block is a voltage limiter that protects the digital processing unit (the STM32F4 Discovery Board) used to acquire the output signal, using its ADC reference voltage as the maximum limit. With the acquired EMG signal, the STM32F4 samples the signal at $1 \mathrm{kHz}$. Then, it is applied a digital $2^{\text {nd }}$ order low-pass Butterworth filter with $1.6 \mathrm{~Hz}$ of cut-off frequency to detect muscle activations, which translates the envelope EMG signal (see Fig.1.B).

\section{Knee Torque Estimation}

We applied the proportional gain method [14] for the knee torque estimation was used forgiven its simplicity and feasibility. It offers a simpler calibration method for clinical applications, due to the smaller number of parameters that need to be tuned and it has a straightforward calibration procedure, when compared to the one that uses the musculoskeletal model.

\section{1) Proportional Gain Method}

The proportional gain method aims to find two constant parameters $\left(K_{f l}, K_{e x}\right)$ that directly transforms EMG signals into knee torque values. The adopted strategy is similar to one reported by [14], which proposed (1) that is equivalent to (2).

$$
\begin{gathered}
\hat{\tau}_{K n e e}=K_{f l} E_{f l}(t)-K_{e x} E_{e x}(t) \\
\Leftrightarrow \hat{\tau}_{\text {Knee }}=\hat{\tau}_{f l}(t)-\hat{\tau}_{e x}(t)
\end{gathered}
$$

In (1), $\hat{\tau}_{\text {Knee }}$ is the estimated knee torque, $E_{f l}(t)$ and $E_{\text {ex }}(t)$ represent the envelope EMG from the flexor and extensor muscles, respectively, and $K_{f l}$ and $K_{e x}$ are the parameters that relate the envelope EMG signals from flexor and extensor muscle, respectively, to the knee torque. Equation (2) is the equivalent of $(1)$, where $\hat{\tau}_{f l}(t)$ is the estimated flexor torque and $\hat{\tau}_{\text {ex }}(t)$ is the estimated extensor torque.

To determine the $K_{f l}$ parameter, it is assumed that a torque being generated by the actuator $\left(\tau_{\text {meas }}\right)$ matches the flexor torque generated by the wearer, $\tau_{f l}(t)$, as (3) shows.

$$
\tau_{\text {meas }}(t)=\tau_{f l}(t)
$$

Considering that the estimated torque provided by the flexor muscles, $\hat{\tau}_{f l}(t)$, attempts to match the torque being generated by the actuator $\left(\tau_{\text {meas }}\right)$, (4) is given.

$$
\hat{\tau}_{f l}(t) \approx \tau_{\text {meas }}(t)
$$

The error between the actuator's torque and the estimated flexor torque can be calculated using (5).

$$
e(k)=\tau_{f l}(k)-\hat{\tau}_{f l}(k)=\tau_{\text {meas }}(k)-\hat{\tau}_{f l}(k)
$$

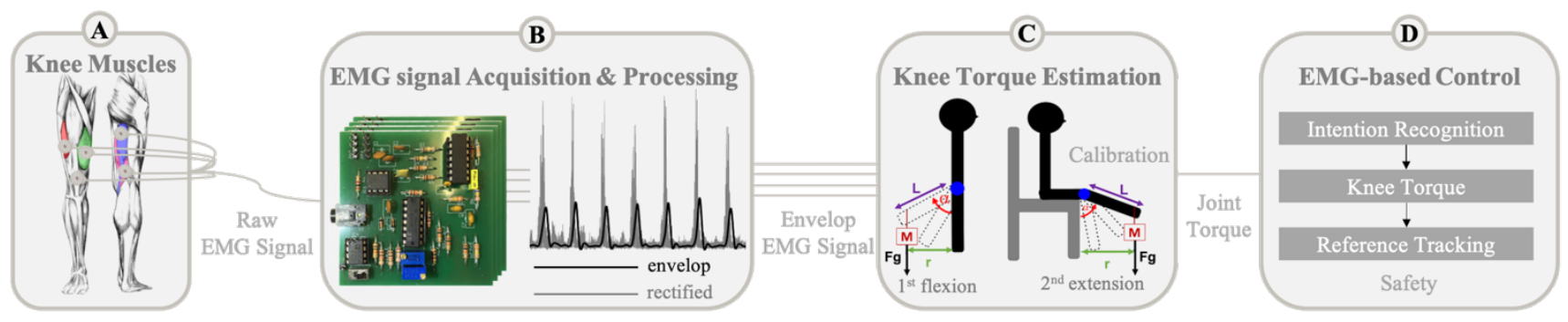

Fig. 1. System Overview. A: chosen muscles to acquire EMG signals, where the extensors marked with red and green are vastus lateralis and vastus medialis, and the flexors marked with purple and pink are semitendinosus and semimembranosus, respectively; B: developed EMG signal acquisition board that provides the rectified raw signal, which is processed to obtain the envelope signal; C: implemented method to estimate knee torque after a calibration routine; D: developed EMG-based control. 
Providing a way to define a performance function based on the error, given by (6).

$$
\begin{gathered}
J=e^{2}(k) \\
=\sum_{k=0}\left(\tau_{\text {meas }}(k)-\hat{\tau}_{f l}(k)\right)^{2} \\
=\sum_{k=0}\left(\tau_{\text {meas }}(k)-K_{f l} E_{f l}(k)\right)^{2}
\end{gathered}
$$

Equation (6) can be minimized by setting its derivative with respect to $K_{f l}$ equal to zero, as shown in (7). Thus, $K_{f l}$ can be calculated by the least square method, given in (8).

$$
\begin{gathered}
\frac{d J}{d K_{f l}}=-2 \sum \tau_{\substack{\text { meas } \\
=0}}(k) E_{f l}(k)+2 K_{f l} \sum E_{f l}^{2}(k) \\
K_{f l}=\frac{\sum \tau_{\text {meas }}(k) E_{f l}(k)}{\sum E_{f l}^{2}(k)}
\end{gathered}
$$

Considering (3), (8) can be rewritten as (9).

$$
K_{f l}=\frac{\sum \tau_{f l}(k) E_{f l}(k)}{\sum E_{f l}^{2}(k)}
$$

The same line of thought is applied to determine $K_{e x}$ parameter. Therefore, the $K_{f l}$ and $K_{e x}$ parameters are determined through an experimental calibration procedure followed by the application of (9).

\section{2) Calibration Procedure}

The development of a new calibration approach oriented to the characteristics of the PKO's actuator is proposed for the computation of $K_{f l}$ and $K_{e x}$ parameters.

The design of the calibration approach considers that the torque value produced by the user's knee $\left(\tau_{\text {Knee }}\right)$ to support a known mass ( $m=$ constant) with the knee at a known angle $(\alpha)$ could be estimated using (10), where $r$ is the knee moment-arm and $F_{\text {Knee }}$ represents the applied force by the user's knee at a given acceleration $(a)$, as stated in (11).

$$
\begin{gathered}
\tau_{\text {Knee }}=F_{\text {Knee }} * r \\
F_{\text {Knee }}=m * a
\end{gathered}
$$

With the user's knee kept still at a known angle $(\alpha=$ constant) and considering that only exists the gravitational acceleration $\left(9.8 \mathrm{~m} / \mathrm{s}^{2}\right),(10)$ can be rewrite as (12).

$$
\tau_{\text {Knee }}=m * 9.8 * r
$$

Measuring the user's leg from the hip to the ground (L) and the angle of the user's knee $(\alpha)$, we can compute the value of $r$, and rewrite (12) as (13).

$$
\tau_{\text {Knee }}=\mathrm{m} * 9.8 * \mathrm{~L} * \sin \alpha
$$

With (13), it is possible to know the user's knee torque to keep the knee still at a given angle and placing electrodes at the selected muscles we can acquire the performed EMG signal that produced that user's knee torque. Considering (9) and (13), the designed calibration approach was developed in two stages, as
Fig.1.C shows. In the first stage, the user was standing with the knee flexed so only the flexion torque is considered $\left(\tau_{f l}\right)$ and in the second stage the user was seated in a chair with the knee extended so only extension torque is considered $\left(\tau_{e x}\right)$. For both stages the applied knee torque is known since the user has to keep the knee still at a known angle with a known mass attached to the foot with surface electrodes placed at the selected muscles. When the expected torque is achieved, the envelope EMG signals from the flexors and extensors are recorded for the computation of the parameters, in accordance with (9), using MATLAB.

\section{EMG-based Control}

As mentioned, the EMG-based control can be classified as a user-oriented assistive strategy. Therefore, its control architecture can follow a hierarchical control architecture [15], as illustrated in Fig.3.

This architecture is organized into three control levels: high, mid- and low-level control. The high-level control infers the user's motion intention from EMG envelopes $\left(E_{f l}(t), E_{e x}(t)\right)$ and includes the calibration procedure, where the found $K_{f l}$ and $K_{\text {ex }}$ parameters during the calibration phase are stored to be applied in the knee joint torque estimation (approached in the mid-level). The mid-level control estimates the knee joint torque using the proportional gain method law, presented in (1). It uses the received EMG envelopes, and the calibrated parameters $K_{f l}$ and $K_{e x}$, for each type of muscles, flexor and extensor, respectively. Subsequently, in the mid-level layer, the reference PKO torque $\left(\tau_{r e f}\right)$ is set equal to the estimated knee joint torque $\left(\hat{\tau}_{\text {Knee }}\right)$. Lastly, the low-level layer implements a torque control through a PID controller law, aiming to track the error $\left(e_{k}\right)$ between the reference knee torque $\left(\tau_{\text {ref }}\right)$ and real torque at the actuator of the knee joint $\left(\tau_{\text {meas }}\right)$, measured by a torque sensor embedded in the PKO, given in (14).

$$
u=K_{p} e_{k}+K_{i} \sum_{n=1}^{k} e_{n} \Delta t+K_{d} \frac{e_{k}-e_{k-1}}{\Delta t}
$$

where $e_{k}$ and $e_{k-1}$ corresponds to the current and previous error between the reference and measured torque value. The Ziegler-Nichols method was applied to find the correct controller gains $\left(K_{p}, K_{i}\right.$, and $\left.K_{d}\right)$, establishing to $K_{p}=90$, and $K_{i}=K_{d}=1.5$.

With respect to the control frequency, the low-level was set to $1 \mathrm{kHz}$ and mid-level to $100 \mathrm{~Hz}$. All control levels run on the STM32F4 digital processing unit. The used PAD, the PKO, is a powered right knee orthosis, a module of the lower limb robotic H2-exoskeleton (Technaid S.L., Spain), a full-body system designed to assist stroke survivors [16]. It has embedded an electronic actuator (DC brushless motor) and sensors (potentiometer, among others). For more details about the hardware and software of the device see [9].

Moreover, to guarantee the safety of the PKO's users, the range of motion of the PKO was limited by software to $10-60$ degrees. This prevents the device from damaging the human lower limbs by applying overextension or over flexion movements, due to small intention (i.e., small muscle contractions) generated near the range of motion limits, that could approach the PKO to its mechanical range of motion 
limits of 0-90 degrees, and the total command signal applied to the PKO passes through a saturator that limits the command to a maximum and minimum value of 2500 and -2500 of pulse width modulation, respectively.

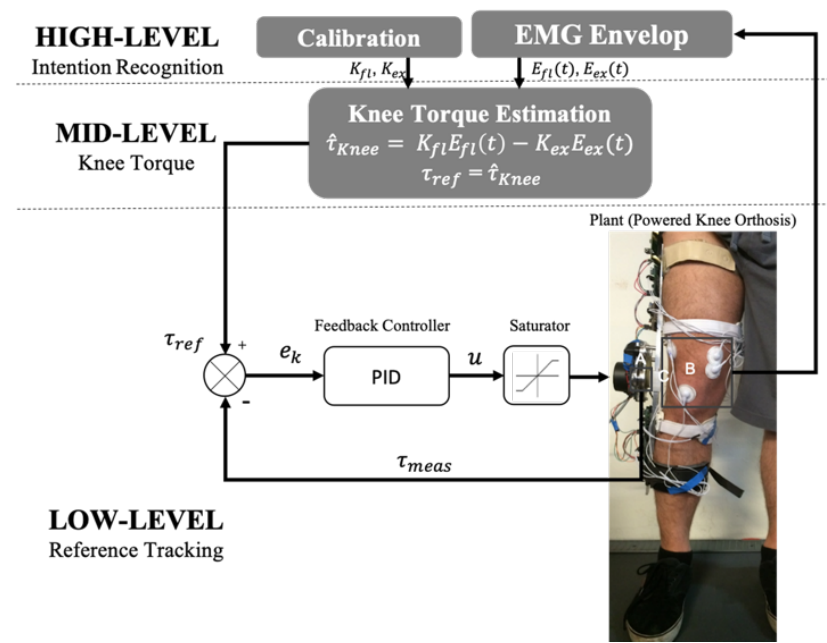

Fig. 2. EMG-based control loop. $E_{f l}(t), E_{e x}(t)$ : envelope EMG signals of flexors and extensors muscles, respectively; $K_{f l}, K_{e x}$ : conversion parameters from envelope EMG signal of flexors and extensors muscles, respectively, into reference knee torque; $\hat{\tau}_{\text {Knee }}:$ reference knee torque; $\tau_{\text {meas }}:$ measured actuator's torque; $e_{k}$ : error between reference knee torque and measured actuator's torque; $u$ : PID command; A: torque sensor; B: surface electrodes; $\mathrm{C}$ : PKO actuator.

\section{VALIDATION}

The overall validation of the control system comprised three phases, as Fig. 3 shows. We included two healthy users (a male and a female) with demographic characteristics of $24.0 \pm 0.0$ years old, height of $1.685 \pm 0.0919 \mathrm{~m}$, and weight of $63.5 \pm$ $14.8492 \mathrm{~kg}$. The electrodes were carefully attached on the 4 selected muscles, Semitendinosus, Semimembranosus, Vastus Medialis and Vastus Lateralis, to collect the raw EMG signals. It was used three surface electrodes for each muscle, which were placed by the same assessor, who carefully followed standard recommendations for surface electrodes assessment [17]. This procedure assures the repeatability of the sensor's placement and minimizes intra-subjects and intra-trials variability. One is a reference electrode that was placed on the center of the knee joint, which is an electrically neutral tissue [18]. The other two electrodes are used to measure the muscle electrical signal and were placed on top of it, separated by about $2 \mathrm{~cm}$ from each other [18]. The possible time-response degradation of surface electrodes over long-term trials did not comprise the bioelectrical activity acquisition given the set short-term duration per session for PKO-based rehabilitation.

Firstly, the calibration procedure (used to compute $K_{f l}$ and $K_{e x}$ ) was validated. The user-specific calibration was valid among difference trials. After a guarantee that the EMG acquisition hardware was properly developed, the amplifier with a variable gain block of the EMG channels for each muscle was tuned regarding the level of muscular activity presented in the user's muscles. This enabled to adjust the system according to the user's muscle condition. The set-up prepared for the experiments is presented in Fig.3.1, for the healthy female. The users were asked to perform isometric contractions during 5 seconds without moving the knee, that enabled to acquire the performed EMG signals. By varying the knee angle $(\alpha)$, it was possible to obtain the intended torques $\left(\tau_{f l} / \tau_{e x}\right)$ at the knee joint, i.e., $8,16,24,32 \mathrm{Nm}$, as these torque values are within the range of PKO's actuator. Table I shows the range of knee joint covered to match the target knee torque and the constant mass and the applied knee force during the calibration procedure. Applying (9), the values of $K_{f l}$ and $K_{e x}$ were found.

TABLE I. Biomechanical values for the calibration procedure.

\begin{tabular}{|c|c|c|c|}
\hline $\begin{array}{c}\text { Knee Joint Angle } \\
\left(\alpha,\left[^{\circ}\right]\right)\end{array}$ & $\begin{array}{c}\text { Torque } \\
\left(\tau_{f l} / \tau_{e x},[\mathrm{Nm}]\right)\end{array}$ & $\begin{array}{c}\text { Mass } \\
(m,[\mathrm{Kg}])\end{array}$ & $\begin{array}{c}\text { Force } \\
\left(F_{\text {Knee }},[\mathrm{N}]\right)\end{array}$ \\
\hline 10.9 & 8 & \multirow{4}{*}{10.7} & \multirow{4}{*}{104.86} \\
\hline 22.4 & 16 & & \\
\hline 34.9 & 24 & & \\
\hline 49.7 & 32 & & \\
\hline
\end{tabular}

The second phase aims to validate the method implemented for the knee joint torque estimation. For this purpose, the subjects were asked to walk in treadmill at different speeds (1 $\mathrm{km} / \mathrm{h}$ and $1.5 \mathrm{~km} / \mathrm{h}$ ) for 3 trails with 3 minutes each, as shown in Fig.3.2. As ground truth, an Inertial Measurement Unit (IMU) was attached to the human shank and thigh, providing in real-time the angle (namely, knee flexion and extension) performed by the user's knee. The two IMUs were integrated in the STM32F4, sampled with $100 \mathrm{~Hz}$ to have all implemented systems synchronized. This procedure has allowed to detect if the muscles were being activated properly through the gait cycle, with the emphasis in the knee flexion and extension. The bioelectrical activity from the muscles was monitored with a sampling frequency of $1 \mathrm{kHz}$.

Lastly, the third phase covers the overall validation of the user-oriented assistive strategy with EMG-based control. It was carried out in treadmill walking for similar speeds (1.0 to 1.5 $\mathrm{km} / \mathrm{h}$ ), as Fig.3.3 displays. During the EMG-based control, the users were asked if the PKO follows their intention to move. The normalized root mean square error (NRMSE) and phase delay between the reference torque $\left(\tau_{\text {ref }}\right)$ and measured torque $\left(\tau_{\text {meas }}\right)$ were inspected. For comparison purposes, a similar trial was conducted with the users wearing the PKO in user-PKO interaction. Each user performed 3 trials for 3 minutes each, for the two control strategies. For both strategies, we collected at $100 \mathrm{~Hz}$ the knee joint angle $(\alpha)$, the estimate torque $\left(\hat{\tau}_{K n e e}\right)$, the PKO torque $\left(\tau_{\text {meas }}\right)$, and the EMG signals $\left(E_{f l}(t), E_{\text {ex }}(t)\right)$.
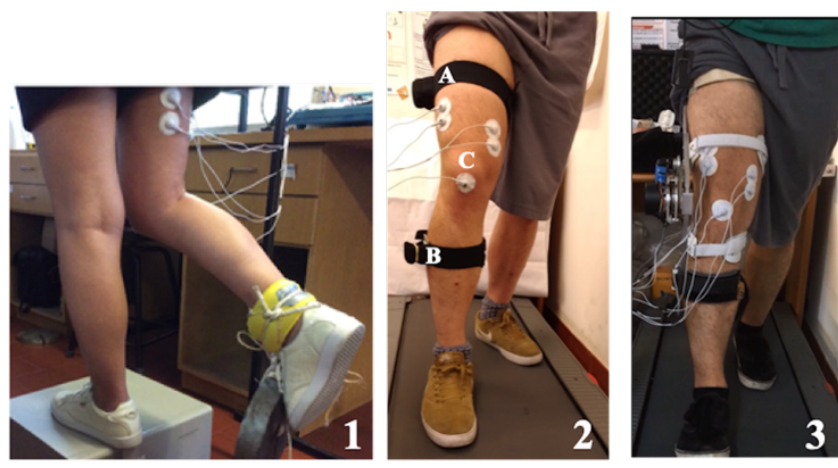

Fig. 3. Performed experimental validations. 1: validation of calibration routine; 2 : validation of knee torque estimation procedure, where $\mathrm{A}$ : thigh IMU; B: shank IMU; C: electrodes 3: validation of user-oriented assistive strategy with EMG-based control. 


\section{RESUlTS \& DisCUSSION}

In a prior validation, we verified that the developed hardware of the EMG acquisition system (green boards in Fig.1.B) shown good performance to acquire EMG signals.

\section{A. Calibration Procedure}

By applying (9), the achieved values of the calibration parameters $K_{f l}$ and $K_{e x}$ for the male subject were 24.3 and 46.7, respectively, and for the female subject, 27.9 and 25.26, respectively. The differences found between the subjects are related with the gain values of the EMG channels used during the EMG acquisition for flexion and extension. For the female, both gains were different while for the male, the gains were very similar. This outcome highlights the needed for a userspecific calibration in an attempt to effectively address a useroriented assistive strategy. Moreover, the envelope of the EMG signals was computed and used to estimate the knee joint torque. Fig.4.A and Fig.4.B present these results. The values of the estimated torques were very similar to the expected ones, as observed in Fig.4.B. This finding suggest that the proposed calibration method was effective for knee torque estimation and relevant to deal with different user's physical conditions. Note that the fluctuations observed in the estimated knee torque (Fig.4.B) resulted from the isometric contraction performed by the user in an attempt to support the selected mass $(m)$ in the defined angle $(\alpha)$. However, this behavior did not affect the performance of the EMG-based control since it was used during dynamic contraction.

\section{B. Knee Torque Estimation}

Fig.5.A suggests that the EMG signals recorded from the 4 muscles are synchronized with the angle of the knee joint. In fact, during the knee flexion, the Semitendinosus and Semimembranosus muscles are activated, and during the knee extension, the extensor muscles, Vastus Medialis and Vastus Lateralis are activated, as expected. Moreover, Fig.5.A also highlights the synchronization of the activation muscles with
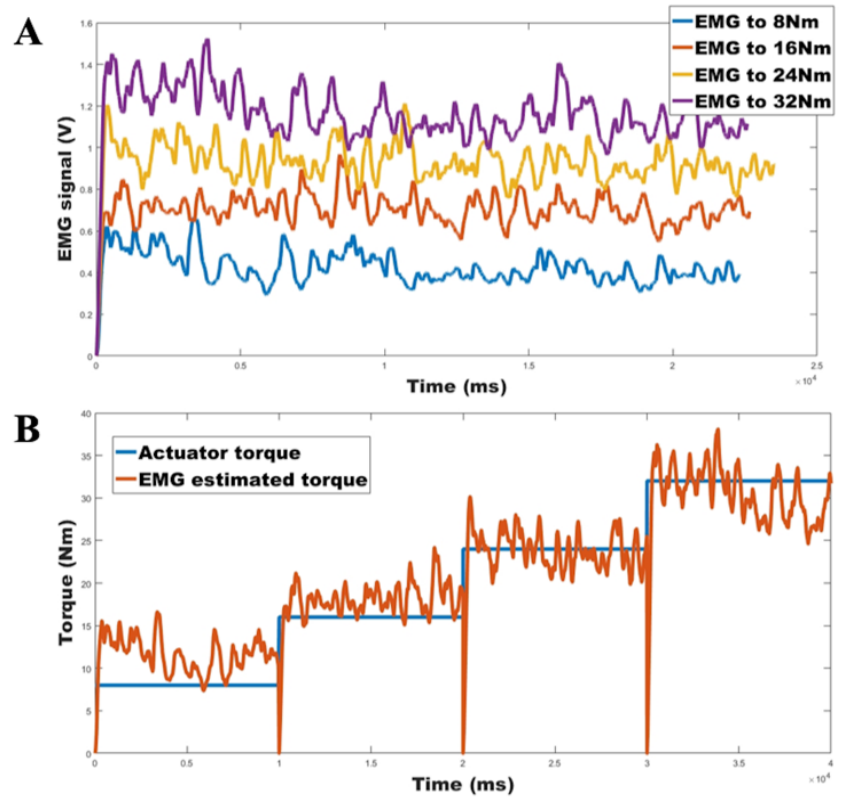

Fig. 4. Results the validation of the calibration procedure from male subject. A: envelop EMG signals; B: Estimated knee torque vs. expected torque. the phases of the human gait: stance (ST), and swing (SW) phases, provided by the IMU signals. Swing phase is mostly described by the knee flexion, and stance phase by the knee extensions [19], [20]. Overall, we can conclude that the results are promising. By inspecting Fig.5.B, we verified that the knee joint torque is being estimated properly. During the knee flexion (angle increases until maximum), the estimated torque also increases positively [21]. When there is an inversion of the limb's excursion (i.e., from flexion to extension, and viceversa), the estimated torque becomes zero at that moment (as illustrates in Fig.5.B as moment reversal). During knee extension (angle decreases until its minimum), the torque decreases [21]. These outcomes show that the torque is being estimated properly, i.e., flexion occurs when positive torques are estimated, and extension occurs when negative torque values are estimated [21]. This is necessary to control the motion with the actuator's torque since this behavior is observed in the actuator of the PKO. These findings report that the performed knee joint torque estimation method was successful and aligned with its low-complexity, it has potential to be used for medical practice.
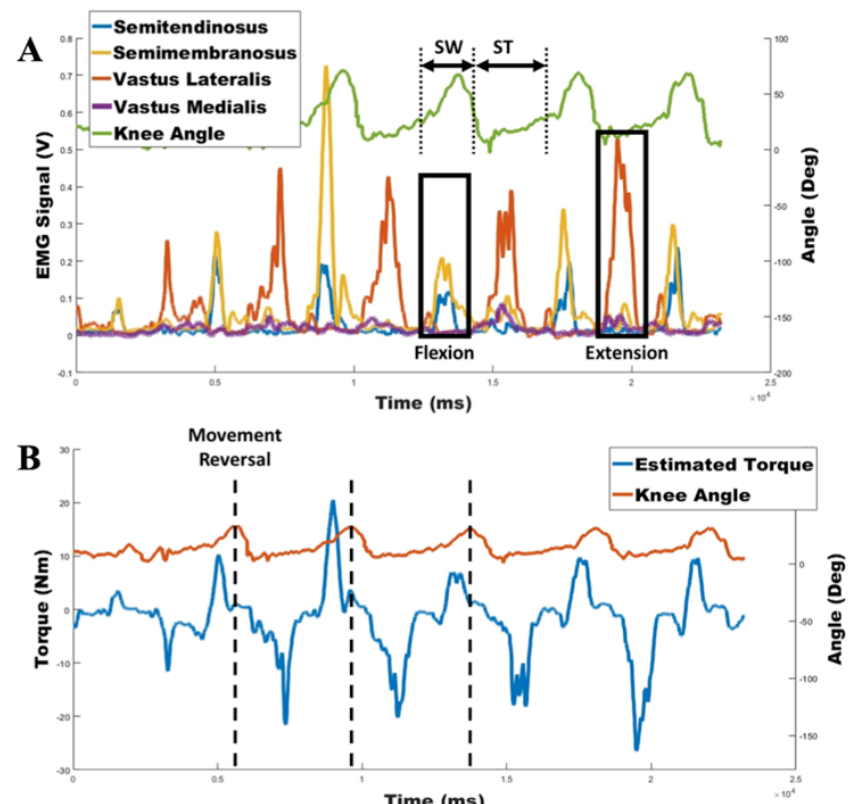

Fig. 5. Results for the validation of the knee torque estimation procedure from a subject walking on treadmill at $1.5 \mathrm{~km} / \mathrm{h}$. A: Envelop EMG signals; B: Estimated torque a measured angle of knee joints. SW: swing phase; ST: stance phase.

\section{EMG-based control vs. User-PKO interaction control}

The results of Fig.6.A show a positive contribution of the EMG-based control. Inspecting the obtained NRMSE, it was achieved a value of $12 \%$ and a phase delay of $22 \mathrm{~ms}$, which are promising results due to low achieved values, concerning we are dealing with a reference that has a period of $3.5 \mathrm{~s}$, approximately. Analyzing Fig.6.B, we observed that the flexor muscles have a mean voltage value of $0.26 \mathrm{~V}$ and the extensor muscle have a mean voltage value of $0.24 \mathrm{~V}$.

To compare the performance of the implemented EMGbased control with another user-oriented strategy, the subjects performed a similar trial with the PKO with User-PKO interaction control. Both are based on user's motion intentions. 

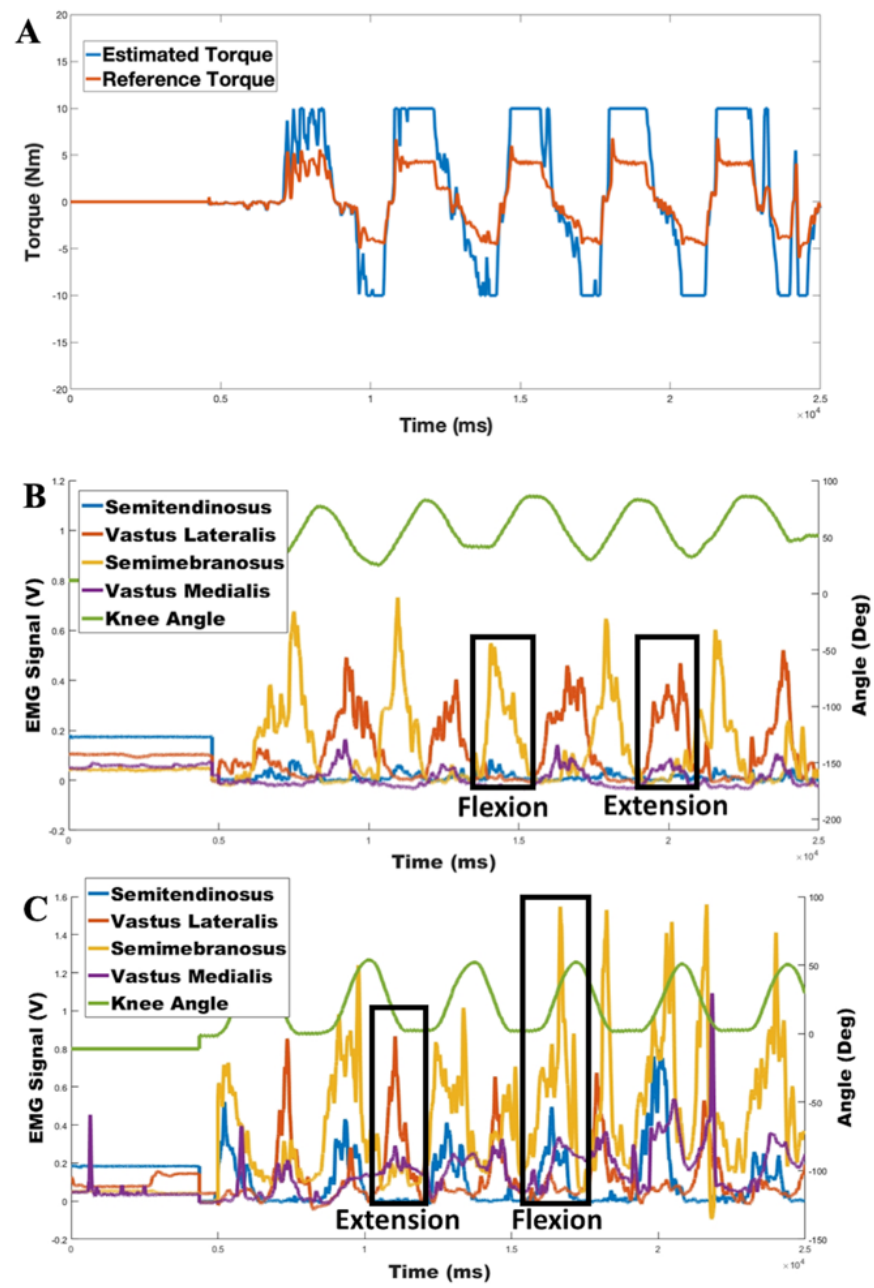

Fig. 6. Results of control strategies of walking experiments in a treadmill at $1 \mathrm{~km} / \mathrm{h}$. A, B: EMG- based control; C: User-PKO interaction control.

However, one reads the User-PKO interaction torque and the other reads the EMG signals. The outcome of this strategy is presented in Fig.6.C. Analyzing the contribution of the muscles to the movement, the flexor muscles have a mean value of 0.54 $\mathrm{V}$ and the extensor muscles have a mean value of $0.35 \mathrm{~V}$. In fact, the outcome of both strategies is in favor of the expected. In User-PKO interaction control, the subject interacts more with the device, and thus, the flexor muscles activation is $52 \%$ higher and the extensor muscles activation is $31 \%$ greater in the UserPKO interaction control. This shows that this strategy is only suitable for patients capable of providing muscular voluntary effort. On the other hand, the EMG-based control requires less effort from the users, suggesting that this strategy is more suitable for subjects with moderate level of impaired gait function in therapies based on the user's intentions. In fact, the subjects reported that their intentions were followed, allowing them to freely move forward. Study limitations comprise the small number of participants for robustness demonstration and the application of musculoskeletal model-based calibration procedures will be more suitable for disabled participants.

\section{CONCLUSION \& FUtURE WORK}

The proposed EMG-based control assistive strategy, based on EMG signals recorded from the thigh muscles, can effectively follow the user's motion intention and may be applied for patients with residual muscular strength. The overall results regarding the EMG-based control proved its wellfunctioning and suitability of the controller for real-time therapies and having time-effective properties due to small phase delay and NRMSE. Future challenges regarding the EMG acquisition system point to the use of wireless technologies to increase the ergonomics of this strategy. In order to achieve more cohesive and decisive results, tests with more participants and walking speeds shall be accomplished.

\section{REFERENCES}

[1] H. Herr, "Exoskeletons and orthoses: classification, design challenges and future directions.," J. Neuroeng. Rehabil., vol. 6, p. 21, 2009.

[2] A. M. Dollar and H. Herr, "Lower Extremity Exoskeletons and Active Orthoses : Challenges and State-of-the-Art.," IEEE Trans. Robot., vol. 24, no. 1 , pp. $144-158,2008$.

[3] C. Fleischer, C. Reinicke, and G. Hommel, "Predicting the Intended Motion with EMG Signals for an Exoskeleton Orthosis Controller," Intell. Robot. Syst., 2005.

[4] W. Hassani, Mohammed, and S. Y. Amirat, "Real-Time EMG driven Lower Limb Actuated Orthosis for Assistance As Needed Movement Strategy," Robot. Sci. Syst., 2013.

[5] C. Fleischer and G.Hommel, "Calibration of an EMG-Based Body Model with six Muscles to control a Leg Exoskeleton," IEEE Int. Conf. Robot. Autom., pp. 10-14, 2007.

[6] H. Kawamoto and Y. Sankai, "Power Assist System HAL-3 for Gait Disorder Person," in International Conference on Computers for Handicapped Persons, 2002, pp. 196-203.

[7] W. Hassani, S. Mohammed, H. Rifa, and Y. Amirat, "EMG Based Approach for Wearer- centered Control of a Knee Joint Actuated Orthosis," Int. Conf. Intell. Robot. Syst., pp. 990-995, 2013.

[8] H. Kawamoto and Y. Sankai, "Power Assist System HAL-3 for Gait Disorder Person," Int. Conf. Comput. Handicap. Pers., pp. 196-203, 2002.

[9] P. Felix, J. Figueiredo, C. P. Santos, and J. C. Moreno, "Eletronic Design and Validation of Powered Knee Orthosis System with Werable Sensors," 17th Int. Conf. Auton. Robot Syst. Compet. (ICARSC 2017), 2017.

[10] C. Fleischer and G. Hommel, "Torque control of an exoskeletal knee with EMG signals," VDI Berichte, 2006.

[11] D. P. Ferris and C. L. Lewis, "Robotic Lower Limb Exoskeletons Using Proportional Myoelectric Control," in 31st Annual International Conference of the IEEE EMBS, 2009, pp. 2119-2124.

[12] C. Fleischer, "Controlling Exoskeletons with EMG signals and a Biomechanical Body Model," 2007.

[13] W. Hassani, "Contribution à la modélisation et à la commande assistive basée intention d' un exosquelette du membre inférieur Walid Hassani Contribution à la modélisation et à la commande assistive basée intention d' un exosquelette du membre," 2015.

[14] G. De Luca, "Fundamental Concepts in EMG Signal Acquisition Table of Contents,", 2003

[15] M. R. Tucker, J. Olivier, A. Pagel, O. Lambercy, and R. Riener, "Control strategies for active lower extremity prosthetics and orthotics : a review," 2015.

[16] M. Bortole et al., "The $\mathrm{H} 2$ robotic exoskeleton for gait rehabilitation after stroke: early findings from a clinical study," J. Neuroeng. Rehabil., vol. 12 , no. 1 , p. $54,2015$.

[17] S. Project, "Recommendations for sensor locations on individual muscles," SENIAM, 2006. [Online]. Available: http://www.seniam.org. [Accessed: 28-Apr-2018].

[18] C. J. De Luca, "Surface Electromyography: Detection and Recording," 2002.

[19] S. J. Piazza, S. L. Delpt, P. Medicine, S. Motor, and P. Program, "The influence of muscles on knee flexion during the swing phase of gait," $J$. Biomech., vol. 29, no. 6, pp. 723-733, 1996.

[20] G. Malanga and J. A. DeLisa, "Clinical Observation," in Gait Analysis in the Science of Rehabilitation, 1998, p. 85.

[21] A. M. El-sayed, N. A. Hamzaid, N. Azuan, and A. Osman, "Technology Efficacy in Active Prosthetic Knees for Transfemoral Amputees: A Technology Quantitative Evaluation,” Sci. World J., p. 17, 2014. 